\title{
Nuclear pairing: Surface or bulk?
}

\author{
N. Sandulescu, ${ }^{1,2}$ P. Schuck, ${ }^{2}$ and X. Viñas ${ }^{3}$ \\ ${ }^{1}$ Institute of Physics and Nuclear Engineering, 76900 Bucharest, Romania \\ ${ }^{2}$ Institut de Physique Nucléaire, Université Paris-Sud, F-91406 Orsay Cedex, France \\ ${ }^{3}$ Departament d'Estructura i Constituents de la Materia, Universitat de Barcelona, Av. Diagonal 647, E-08028 Barcelona, Spain
}

(Received 5 January 2005; published 11 May 2005)

\begin{abstract}
We analyze how the spatial localization properties of pairing correlations are changing in a major neutron shell of heavy nuclei. It is shown that the radial distribution of the pairing density depends strongly on whether the chemical potential is close to a low or a high angular momentum level and has little sensitivity to whether the pairing force acts at the surface or in the bulk. The pairing density averaged over one major shell is, however, rather flat, exhibiting little dependence on the pairing force. Hartree-Fock-Bogoliubov calculations for the isotopic chain ${ }^{100-132} \mathrm{Sn}$ are presented for demonstration purposes.
\end{abstract}

DOI: 10.1103/PhysRevC.71.054303

PACS number(s): 21.60.-n, 21.10.-k

\section{INTRODUCTION}

There is an ongoing debate whether pairing in nuclei is concentrated preferentially in the bulk or at the surface. Quite a few authors plead for a surface enhancement of nuclear pairing [1,2]. This belief is based mostly on two arguments. First, pairing is concentrated around the Fermi energy and, in heavy nuclei, levels at the Fermi energy are dominated by high $l$ values with corresponding wave functions peaked at the surface. Second, the idea of surface dominance of pairing was probably mostly fostered from the local density approximation (LDA) picture. Indeed, from Fig. 3 of Ref. [3], where the neutron gap at the Fermi surface is shown in symmetric nuclear matter as a function of $k_{F}$, calculated once with a realistic bare force and once with the Gogny D1S force, one can see that, adopting the LDA, the gap in the interior of a nucleus should be very small, whereas at the surface it should pass through a huge peak over $2.0 \mathrm{MeV}$ high. In contrast, using LDA one obtains on average a reasonable agreement for the correlation energy in comparison with quantal calculations [4]. However, to conclude from this fact that the LDA also provides a good approximation for the $r$ dependence of $\Delta$ in nuclei is a little premature since one knows that Thomas-Fermi theory, on which LDA is based, yields local quantities that must be interpreted as distributions, which are useful under integration but locally often quite erroneous [5].

Actually, the two arguments presented here are related to two different aspects of pairing in finite nuclei. Thus, the first argument refers to the radial distribution of pairing correlations (i.e., of Cooper pairs formed upon various singleparticle states). Commonly, the spatial localization of pairing correlations is described by the pairing density. In finite nuclei the pairing density is ascribed to the radial form factor of pair transfer reactions [6-8]. These reactions probe the strength of pairing correlations as manifested in pair-vibration and pair-rotation modes [9]. In contrast, the pairing field $\Delta(r)$, on which the second argument is based, provides not only informations about the localization of pairing correlations but also on the properties of the effective pairing force. This is evident from the fact that, by definition, $\Delta(r)$ is given by the convolution between the pairing force and the pairing density.
As will be shown in this paper, in finite nuclei the radial dependence of the pairing density is rather insensitive to the type of the pairing force. Consequently, in finite nuclei $\Delta(r)$ and the associated global quantities (e.g., pairing gaps) carry in fact information related essentially to the effective paring force and much less on the localization of Cooper pairs.

An earlier indication that pairing correlations may not be tremendously surface peaked came from the study of pairing density in half-infinite nuclear matter with the Gogny force [10]. There, the peaking of the pairing density at the surface was only very moderate. From Fig. 1 of Ref. [10] it is even not evident whether the peaking is a pure consequence of Friedel oscillations [11]. For finite nuclei only very few calculations of the pairing density as a function of the radius are available [12]. These calculations mainly focus on how the pairing density changes from one major shell to the other, up to the neutron drip line. The scope of the present paper is to give a more systematic investigation of the localization properties of the pairing density in one major shell. Thus, it will be shown that the bulk versus surface localization of pairing correlations can change significantly in only one major shell, depending on whether the chemical potential is close to a low or a high angular momentum level. This behavior is illustrated here for the chain of $\mathrm{Sn}$ isotopes with neutron number between $N=50$ and $N=82$. The study is performed in the Hartree-FockBogoliubov (HFB) approach and using surface versus bulkdominated pairing interactions. It then will be argued that only an average over one major shell makes sense to reveal the generic features of nuclear pairing with respect to the surface and/or the bulk. The general framework of the calculations is described in Sec. II. Then, in Sec. III, we discuss the radial distribution of pairing correlations as provided by the HFB calculations. Finally, in Sec. IV we present our conclusions.

\section{FORMALISM: HARTREE-FOCK-BOGOLIUBOV APPROACH}

In superfluid Fermi liquids, pairing correlations are usually characterized by the "condensate" wave function [13]. For the case of ${ }^{1} S_{0}$ pairing the condensate wave function, referred to 
here as the pairing density, is given by

$$
\kappa(\mathbf{r})=\langle\psi(\mathbf{r}, s=1 / 2) \psi(\mathbf{r}, s=-1 / 2)\rangle,
$$

where the operator $\psi(\mathbf{r}, s=1 / 2)$ annihilates a nucleon at the point $\mathbf{r}$ and with the spin projection $s=1 / 2$. This local pairing density describes the center-of-mass distribution of the Cooper pairs inside the superfluid. For translationally invariant superfluid systems at zero temperature, $\kappa(\mathbf{r})$ is a constant field with the modulus equal to $\sqrt{n}_{0}$, where $n_{0}$ is the number of the condensed (Cooper) pairs [13]. In large superfluid systems, in which the condensate is macroscopically occupied, $\kappa(\mathbf{r})$ can still be regarded as a classical coherent field with negliglibe variations over distances greater than the coherence length. However, when the range of pairing correlations (associated with the mean-square radius of Cooper pairs) is comparable with the size of the system, as happens in finite nuclei, the local variations of the pairing density are important and should be calculated from a quantum equation of motion of the Bogoliubov-de Gennes type [14]. In finite nuclei for such a task one usually employs the HFB equations [5], in which both the mean field and the pairing field are calculated self-consistently.

In this study the HFB equations are solved by imposing spherical symmetry and employing zero-range forces in both the particle-hole and particle-particle channels. Under these conditions the radial HFB equations have the following form:

$$
\left(\begin{array}{cc}
h(r)-\lambda & \Delta(r) \\
\Delta(r) & -h(r)+\lambda
\end{array}\right)\left(\begin{array}{c}
\mathfrak{U}_{i}(r) \\
\mathfrak{V}_{i}(r)
\end{array}\right)=E_{i}\left(\begin{array}{c}
\mathfrak{U}_{i}(r) \\
\mathfrak{V}_{i}(r)
\end{array}\right),
$$

where $h(r)$ and $\Delta(r)$ are, respectively, the mean-field Hamiltonian and pairing field, and $\lambda$ is the chemical potential. All basic quantities such as particle density $\rho(r)$ and pairing density $\kappa(r)$ are expressed in terms of the upper and lower components of the HFB wave function. Thus,

$$
\begin{aligned}
\rho(r) & =\frac{1}{4 \pi} \sum_{i}\left(2 j_{i}+1\right) \mathfrak{V}_{i}^{*}(r) \mathfrak{V}_{i}(r), \\
\kappa(r) & =\frac{1}{4 \pi} \sum_{i}\left(2 j_{i}+1\right) \mathfrak{U}_{i}^{*}(r) \mathfrak{V}_{i}(r) .
\end{aligned}
$$

In the present HFB calculations we use in the particle-hole channel a Skyrme-type force (i.e., SLy4 [15]). Hence the mean field has the standard expression in terms of single-particle densities $[16,17]$. For the pairing interaction we use two forces, that is, a pure $\delta$ force and a density-dependent $\delta$ (DDD) force. For the latter we take the form [18]

$$
\begin{aligned}
V\left(\mathbf{r}-\mathbf{r}^{\prime}\right) & =V_{0}\left[1-\eta\left(\frac{\rho}{\rho_{0}}\right)^{\alpha}\right] \delta\left(\mathbf{r}-\mathbf{r}^{\prime}\right) \\
& \equiv V_{\mathrm{eff}}(\rho(r)) \delta\left(\mathbf{r}-\mathbf{r}^{\prime}\right) .
\end{aligned}
$$

The pairing interaction acts upon the pairing density through the pairing field, which for the DDD force is given by

$$
\Delta(r)=\frac{V_{\text {eff }}}{2} \kappa(r) .
$$

Because of the divergences associated with a zero-range pairing force, the HFB calculations should be performed with an energy cutoff. The energy cutoff and the strength of the interaction can be eventually related to each other
TABLE I. Results of HFB calculations for Sn isotopes obtained with the DDD force. $A$ is the atomic mass, $\lambda$ is the chemical potential (in $\mathrm{MeV}$ ), and $\langle\Delta\rangle$ is the averaged pairing gap (in $\mathrm{MeV}$ ). The latter is calculated by convoluting the pairing field with the pairing density. The next five rows give the occupation probabilities for the singleparticle states of the valence shell. Shown in parentheses for each of these states is the Hartree-Fock energy corresponding to the midshell isotope ${ }^{116} \mathrm{Sn}$.

\begin{tabular}{lcrrrrr}
\hline \hline $\mathrm{A}$ & 104 & 108 & 114 & 116 & 124 & 128 \\
$\lambda$ & -10.9 & -10.0 & -8.97 & -8.64 & -7.3 & -6.7 \\
$\langle\Delta\rangle$ & 1.4 & 1.77 & 1.91 & 1.88 & 1.6 & 1.3 \\
$2 d_{5 / 2}(-10.1)$ & 0.63 & 0.73 & 0.88 & 0.91 & 0.97 & 0.98 \\
$1 g_{7 / 2}(-9.7)$ & 0.17 & 0.28 & 0.65 & 0.74 & 0.94 & 0.97 \\
$3 s_{1 / 2}(-8.8)$ & 0.097 & 0.16 & 0.43 & 0.54 & 0.89 & 0.95 \\
$2 d_{3 / 2}(-8.3)$ & 0.07 & 0.11 & 0.30 & 0.4 & 0.83 & 0.93 \\
$1 h_{11 / 2}(-6.7)$ & 0.03 & 0.05 & 0.11 & 0.14 & 0.44 & 0.7 \\
\hline \hline
\end{tabular}

through the scattering length of the di-neutron system [18] or by using more sophisticated regularization procedures [19]. In the present calculations we use an energy cutoff (in the quasiparticle spectrum) equal to $60 \mathrm{MeV}$. The strength of the DDD force is taken equal to $V_{0}=-430 \mathrm{MeV} \mathrm{fm}^{-3}$; for the parameters that set the density dependence of the DDD force we use the same values as in Ref. [3] (i.e., $\eta=0.45$ and $\alpha=0.47)$. This parametrization assures reasonable gap values in Sn isotopes. It is worth mentioning that in Ref. [3] the last two parameters (i.e., the ones that fix the density dependence of the DDD force) were fitted to reproduce the density dependence of pairing gap in nuclear matter provided by the Gogny D1S force. Thus, from this perspective, a finiterange density-independent Gogny D1S force is equivalent to a DDD force that, according to the terminology used in Refs. [20,21], is a mixture between a volume and a surface pairing interaction. In the HFB calculations with the pure $\delta$ force we have taken a strength $V_{0}=-220 \mathrm{MeV} \mathrm{fm}^{-3}$. With this value of the strength we get for $\mathrm{Sn}$ isotopes approximately the same pairing energies as for the DDD force.

\section{RESULTS: PAIRING LOCALIZATION IN Sn ISOTOPES}

Before we start analyzing the local properties of pairing correlations, we first present the global quantities that charaterize the HFB solution. Thus, Table I shows how the chemical potential $\lambda$, the averaged gaps, and the occupation probabilities of single-particle states are evolving by filling the major shell $N=50-82$. Changes of particle density with neutron number are shown in Fig. 1(a). These changes can be easily traced back to the occupation probabilities of single-particle wave functions shown in Fig. 1(b). Thus, one notices the progressive increase of the particle density at small distances, produced by the filling of the state $3 s_{1 / 2}$.

Next we discuss how the localization properties of the pairing density change with the filling of the major shell $N=50-82$. The results for $\kappa(r)$ obtained by using the two zero-range pairing forces introduced in the previous section are shown in Fig. 2. 

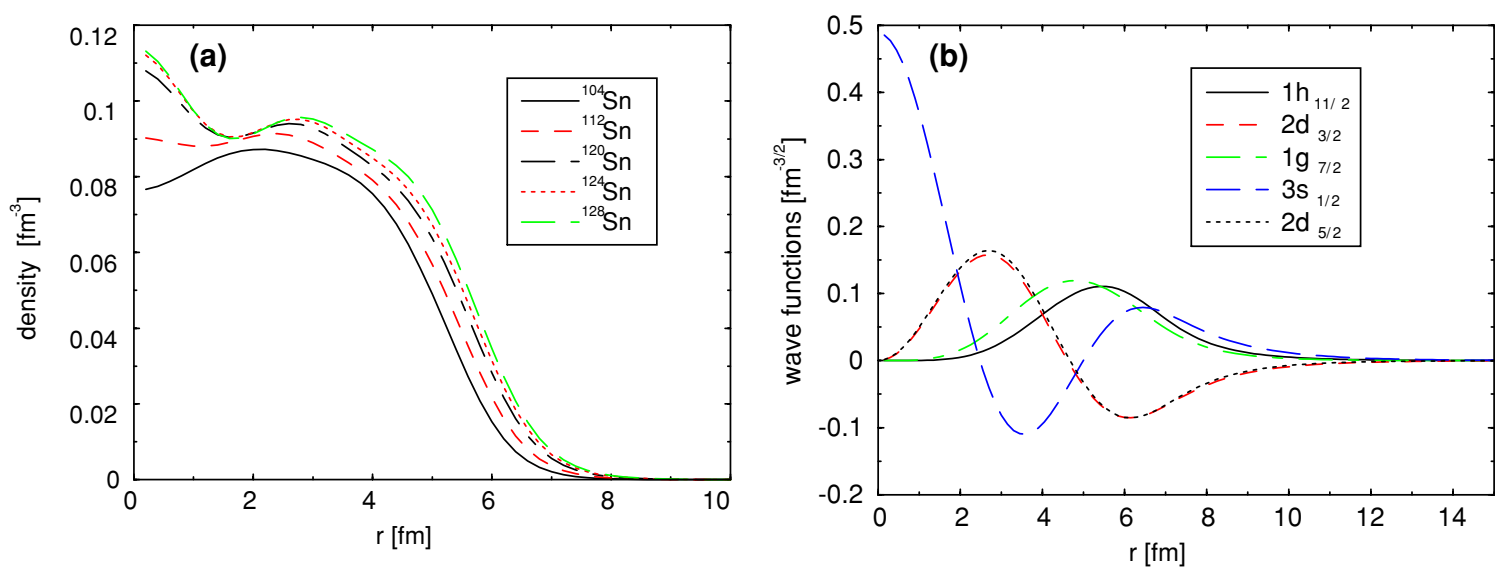

FIG. 1. (Color online) The particle density (a) for various Sn isotopes and the Hartree-Fock wave functions (b) corresponding to the valence shell of ${ }^{116} \mathrm{Sn}$.

First, we notice that for both pairing forces $\kappa(r)$ changes quite strongly from $A=104$ to $A=128$. Thus, we see that at the beginning of the shell, when the contribution of the states $2 d_{5 / 2}$ and $1 g_{7 / 2}$ is dominant, the pairing density is slightly larger inside the nucleus than at the surface. Then, at midshell, the pairing density becomes on average almost flat for an extended region from about 1 to $5 \mathrm{fm}$. We also notice a large bump close to the center of the nucleus produced by the neutrons distributed in the state $3 s_{1 / 2}$.

In the second half of the major shell the chemical potential becomes closer to the intruder state $1 h_{11 / 2}$, which starts progressively to dominate the structure of $\kappa(r)$. Since the wave function of $1 h_{11 / 2}$ is localized in the surface region [see Fig. 1(b)], the pairing density also gets a bump around $5 \mathrm{fm}$. This bump becomes more pronounced toward the end of the shell. However, one can see that toward the end of the shell the pairing density decreases rather strongly in the center of the nucleus. This behavior reflects the smaller contribution of the state $3 s_{1 / 2}$ toward $N=82$.

A somewhat surprising but important finding, shown in Fig. 2, is that the radial structure of $\kappa(r)$ changes very little with the density dependence of the $\delta$ force. Through this dependence the strength of the pairing force is reduced inside the nucleus compared to the surface region. Consequently, the relative contribution of the state $3 s_{1 / 2}$ to pairing correlations is suppressed. However, because of its small degeneracy compared to the other states, this suppression has no important consequences on $\kappa(r)$, as can be seen in Fig. 2.

As we have seen, the radial dependence of the pairing density in $\mathrm{Sn}$ isotopes is dominated by the individual shell structure. To reveal a generic behavior one should follow the old idea of Strutinsky [22] and average the pairing density over one major shell. Here we have taken the crude arithmetic average over all even $\mathrm{Sn}$ isotopes, leaving the study of more refined averaging methods for future work. The result is shown in Fig. 3. We remark that on average the pairing density $\bar{\kappa}(r)$ is quite flat over the bulk. Since we are considering an even-parity shell, we have at the origin a quite pronounced bump from the s wave. Had we considered an odd-parity shell, we certainly would see a corresponding hole at the origin [23]. In Fig. 3 we also see that despite the fact that the densitydependent force yields, with respect to the pure $\delta$ force, slight surface enhancement and volume depression, the difference
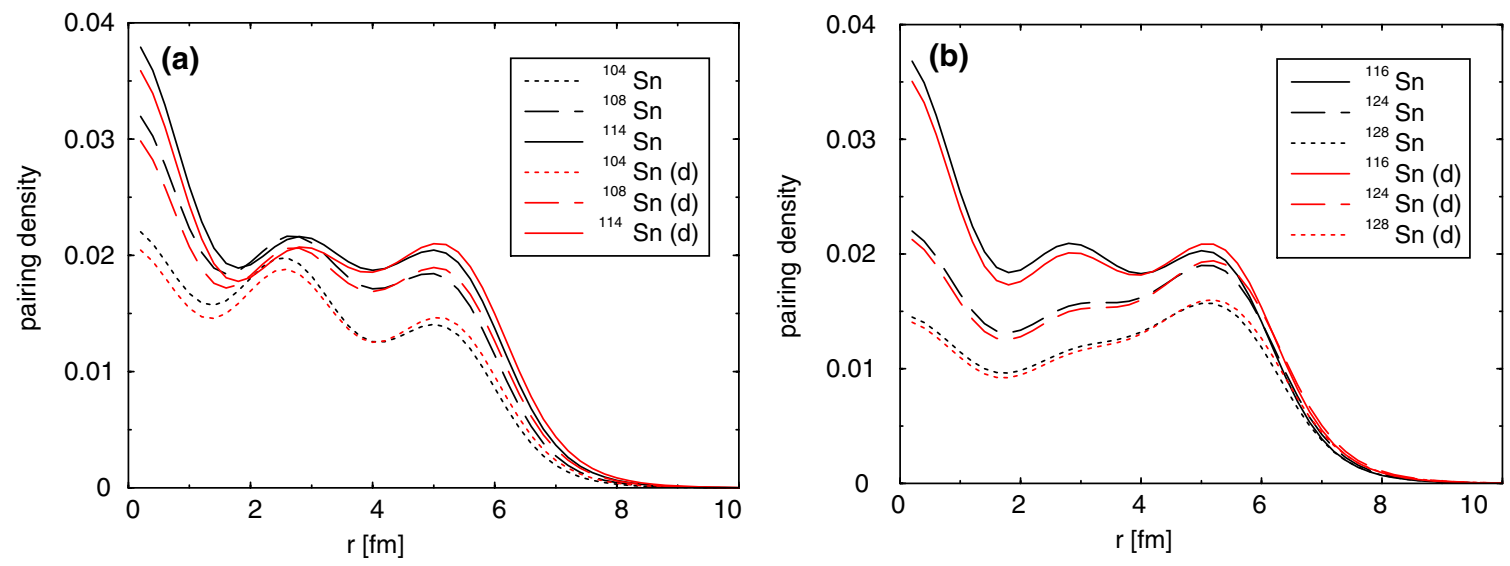

FIG. 2. (Color online) Neutron pairing densities $\kappa(r)\left(\right.$ in $\mathrm{fm}^{-3}$ ) for Sn isotopes calculated in the HFB approach. The black (red) curves correspond to the $\delta$ force (density-dependent $\delta$ force). 


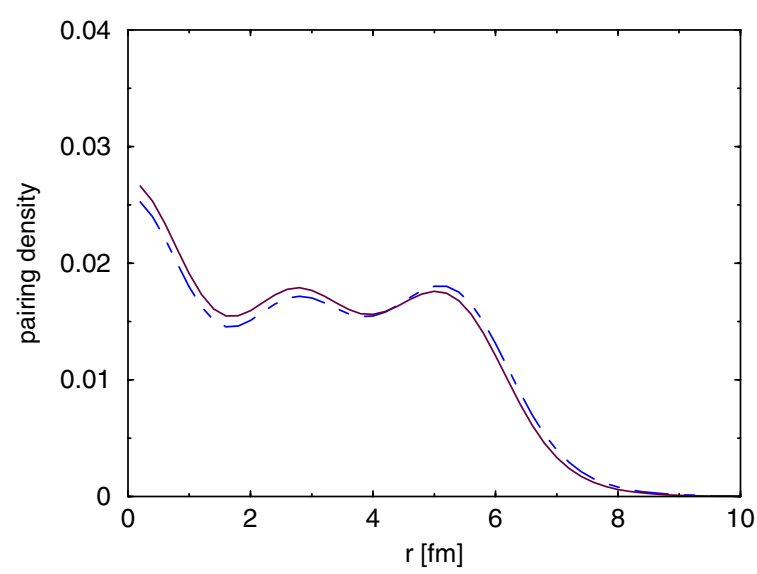

FIG. 3. (Color online) Averaged pairing density (in $\mathrm{fm}^{-3}$ ) calculated in the HFB approach. The solid (dot-dashed) curve corresponds to $\delta$ force (density-dependent $\delta$ force).

induced upon $\bar{\kappa}(r)$ by the two pairing forces is practically insignificant.

To further understand what happens, let us take the BCS approximation of $\kappa$ and replace, for the midshell situation, the $u v$ factors with a unique constant equal to one, that is, $\kappa(r) \approx$ $\frac{1}{4 \pi} \sum_{i} u_{i} v_{i}\left|\phi_{i}(r)\right|^{2} \approx \frac{1}{4 \pi} \sum_{i}^{\prime}\left|\phi_{i}(r)\right|^{2}$, where the prime indicates that the sum runs over the major shell only and $\phi_{i}(r)$ are the single-particle wave functions. Taking the wave functions plotted in Fig. 1(b) for $\phi_{i}(r)$, one gets for $\kappa$ the result shown in Fig. 4. By summing in $\kappa$ all single-particle states with the same $u v$ factor one overestimates the contribution of those single-particle states that are far from the chemical potential, mainly of the states $2 d_{5 / 2}$ and $h_{11 / 2}$. This fact produces the extended tail seen in Fig. 4. Apart from that, we can see that this rough approximation of $\kappa$ follows rather well the flat radial structure of the HFB curve.

A strong dependence of the pairing density profile on the individual shell structure can be clearly observed even for a pairing force that is drastically reduced inside the nucleus. The results for such a pairing force, with the parameters $\eta=1$,

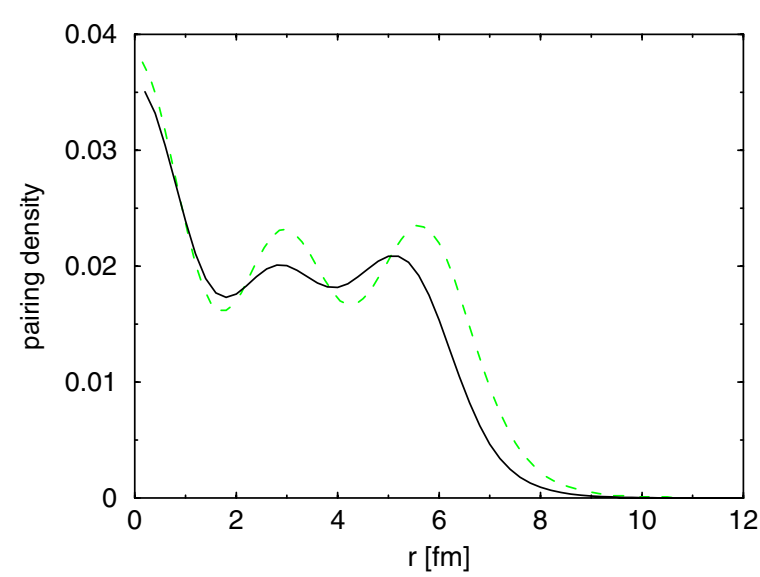

FIG. 4. (Color online) Pairing density in ${ }^{116} \mathrm{Sn}$. The solid line is the HFB result. The dotted line corresponds to the summation of the square of single-particle wave functions (see text).

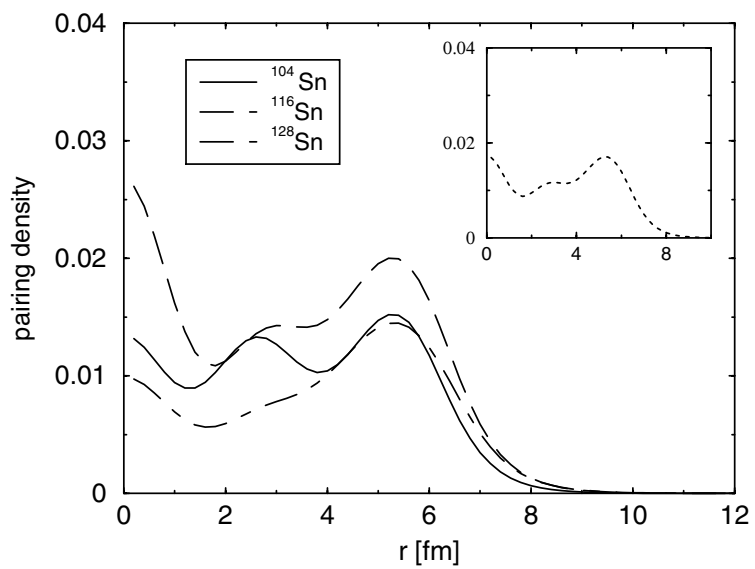

FIG. 5. Neutron pairing densities $\left(\right.$ in $^{-3} \mathrm{fm}^{-3}$ ) for several $\mathrm{Sn}$ isotopes calculated by using a DDD force with $\eta=1$. The inset contains the average value of the pairing density.

$\alpha=1$, and $V_{0}=600 \mathrm{MeV} \mathrm{fm}^{-3}$, are shown in Fig. 5. This pairing force gives a pairing field that is almost entirely concentrated in the surface region (see Fig. 6). However, in spite of this very strong surface localization of the pairing field, the pairing densities shown in Fig. 5 still have a large volume component, especially for ${ }^{104} \mathrm{Sn}$, which changes significantly with neutron number. This also can be seen for the average pairing density (see the inset in Fig. 5).

From all these results it appears that in a particular nucleus the radial distribution of the pairing correlations, given by the pairing density, depends strongly on the localization of single-particle states that are close to the chemical potential and much less on the type of pairing force. The dependence of pairing density profile on the filling of the major shells can be eventually checked in pair-transfer reactions.

The quantity that depends strongly on the assumption made on the type of pairing force is the pairing field. This dependence is clearly seen in Fig. 6, where the averaged pairing fields corresponding to the $\delta$ force and the DDD forces are plotted. Hence, indications about which type of pairing force might be

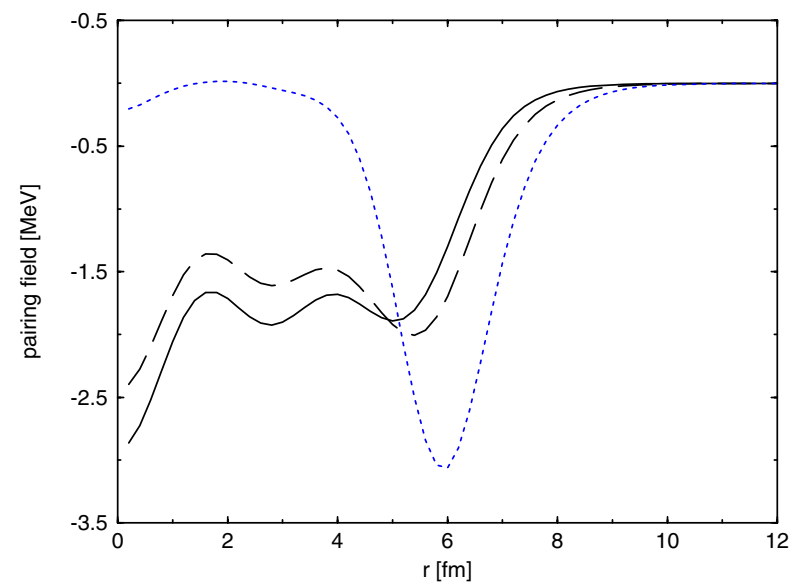

FIG. 6. (Color online) Averaged pairing field (in $\mathrm{MeV}$ ) for $\mathrm{Sn}$ isotopes. The solid curve corresponds to the $\delta$ force; the dashed (dotted) lines are the results for the DDD force with $\eta=0.45(\eta=1)$. 
more appropiate in finite nuclei can eventually be extracted from quantities related to the pairing field (e.g., the pairing gaps). Thus, according to Ref. [20] a DDD force with $\eta=1 / 2$ and $\alpha=1$ would give better results for the odd-even mass staggering than a $\delta$ force or a DD force with $\eta=1$ and $\alpha=1$. However, a pure $\delta$ force gives for the one- and the two-neutron separation energies almost the same results as a DDD force [24]. Therefore, from these calculations one cannot yet draw a definite conclusion on how much stronger the pairing force should be at the surface compared to the bulk. Nevertheless, the fact that the pairing force should have some surface peaking is suggested by all bare interactions, which are more attractive for small momenta than for large ones (see also the $V_{\text {low-k }}$ force based on realistic bare nucleon-nucleon interactions $[25,26])$. However, bare forces do not yield enough pairing in finite nuclei [27] and additional attraction from the induced interaction (phonons) may still enhance the surface behavior of the effective pairing force [28].

\section{SUMMARY AND CONCLUSIONS}

In this work we have investigated the radial distribution of pairing correlations in the major neutron shell $N=50-82$ of $\mathrm{Sn}$ isotopes. The localization of pairing correlations, given by the pairing density, is calculated in the framework of the HFB approach using two effecive pairing interactions: a pure $\delta$ force and a $\delta$ force with a density-dependent factor that enhances the strength of the interaction at low density (i.e., in the surface region). It was found that the pairing density changes strongly going from one end to the other of the major shell $N=50-82$, depending on whether the chemical potential is close to a level with low or high $l$ value. However, the differences with respect to the two pairing forces remained insignificant.
Then, to obtain some generic behavior of the pairing density $\kappa$ in nuclei, we performed an average of $\kappa$ 's over one major shell. The resulting averaged pairing density $(\bar{\kappa})$ is, apart from some oscillations, practically constant over the whole volume except for a quite pronounced peak at the origin, produced by the $3 s_{1 / 2}$ state contained in the open major shell of Sn isotopes. The results for $\bar{\kappa}$ show again very little sensitivity to the type of pairing force used in the calculations, with the exception of the extreme case where the pair field is completely suppressed in the interior of the nucleus.

We also have calculated the corresponding pairing fields $\Delta(r)$. By definition the pairing field is directly proportional to the pairing force [see Eq. (6)]. Thus, even if for a given DDD force the pairing density is almost constant over the nuclear volume, the corresponding pairing field shows a surface enhancement similar to that of the DDD force. This is clearly seen in Fig. 6. Therefore in finite nuclei quantities relating to the radial distribution of the pairing field (e.g., odd-even mass differences) probe the density dependence of zero-range pairing forces rather than the localization of pairing correlations (i.e., of Cooper pairs).

In summary, the radial distribution of pairing correlations in finite nuclei depends strongly on the localization of single-particle states that are closest to the chemical potential and much less on the surface or bulk enhancement of the pairing force. However, to reveal generic features an average over the shell fluctuations should be taken. This reveals that even strongly surface peaked pairing fields $\Delta(r)$ induce only a moderate surface enhancement of the local pairing density $\kappa(r)$.

The conclusions of this study are based on zero-range pairing forces. However, the results with a finite-range force of the Gogny type, which will be published in a future paper together with a semiclassical study of nuclear pairing localization [29], show the same trends.
[1] N. Tajima, P. Bonche, H. Flocard, P.-H. Heenen, and M. S. Weiss, Nucl. Phys. A551, 434 (1993).

[2] S. A. Fayans, S. V. Tolokonnikov, E. L. Trykov, and D. Zawischa, Nucl. Phys. A676, 49 (2000).

[3] E. Garrido, P. Sarriguren, E. Moya de Guerra, and P. Schuck, Phys. Rev. C 60, 064312 (1999).

[4] H. Kucharek, P. Ring, P. Schuck, R. Bengtsson, and M. Girod, Phys. Lett. B216, 249 (1989).

[5] P. Ring and P. Schuck, The Nuclear Many-Body Problem (Springer-Verlag, Berlin, 1980).

[6] A. Bohr and B. Mottelson, Nuclear Structure (Benjamin, New York, 1969).

[7] W. von Oertzen and A. Vitturi, Rep. Prog. Phys. 64, 1247 (2001).

[8] E. Khan, N. Sandulescu, N. V. Giai, and M. Grasso, Phys. Rev. C 69, 014314 (2004).

[9] R. A. Broglia, O. Hansen, and C. Riedel, Adv. Nucl. Phys. 6, 287 (1973).

[10] M. Farine and P. Schuck, Phys. Lett. B459, 444 (1999).

[11] J. Friedel, Nuovo Cimento Suppl. 7, 287 (1958).

[12] J. Dobaczewski, W. Nazarewicz, T. R. Werner, J. F. Berger, C. R. Chinn, and J. Decharge, Phys. Rev. C 53, 2809
(1996); J. Dobaczewski et al., Nucl. Phys. A693, 361 (2001).

[13] P. Noziere and D. Pine, The Theory of Quantum Liquids (Addison-Wesley, Reading, MA, 1990).

[14] P. G. de Gennes, Superconductivity of Metals and Alloys (Addison-Wesley, Reading, MA, 1989).

[15] E. Chabanat, P. Bonche, P. Haensel, J. Meyer, and R. Schaeffer, Nucl. Phys. A635, 231 (1998).

[16] D. Vautherin, and D. M. Brink, Phys. Rev. C 5, 626 (1972).

[17] J. Dobaczewski, H. Flocard, and J. Treiner, Nucl. Phys. A422, 103 (1984).

[18] G. F. Bertsch and H. Esbensen, Ann. Phys. (NY) 209, 327 (1991).

[19] A. Bulgac and Yongle Yu, Phys. Rev. Lett. 88, 042504 (2002).

[20] J. Dobaczewski, W. Nazarewicz, and M. V. Stoitsov, nuclth/0109073.

[21] J. Dobaczewski, W. Nazarewicz, and M. V. Stoitsov, Eur. Phys. J. A 15, 21 (2002).

[22] V. M. Strutinsky, Nucl. Phys. A95, 420 (1967).

[23] X. Vinas, P. Schuck, M. Farine, and M. Centelles, Phys. Rev. C 67, 054307 (2003). 
[24] Yongle Yu and A. Bulgac, Phys. Rev. Lett. 90, 222501 (2003); nucl-th/0210047.

[25] S. K. Bogner, T. T. S. Kuo, A. Schwenk, D. R. Entem, and R. Machleidt, Phys. Lett. B576, 265 (2003).

[26] A. Sedrakian, T. T. S. Kuo, H. Müther, and P. Schuck, Phys. Lett. B576, 68 (2003).
[27] F. Barranco, R. A. Broglia, G. Colo, E. Vigezzi, and P. F. Bortignon, Eur. Phys. J A 21, 37 (2004).

[28] F. Barranco, P. F. Bortignon, R. A. Broglia, G. Colo, P. Schuck, E. Vigezzi, and X. Viñas, nucl-th/0502076.

[29] E. Vigezzi et al. (in preparation). 